\title{
Sistema Portátil para Medida On-line da Distribuição de Tempo de Residência na Extrusão
}

\author{
Denilson M. Santos e Sebastião V. Canevarolo Jr
}

Resumo: A Curva de Distribuição de Tempos de Residência - CDTR tem sido largamente usada para caracterização do tipo de fluxo em extrusoras, sendo que medidas "on-line" permitem diagnosticar de modo muito rápido problemas como desgaste entre a rosca e o barril da extrusora, estagnação do material e outros. A CDTR pode ser determinada fazendo-se uso da técnica de estímulo de pulso único, onde um traçador é introduzido no sistema em um dado instante e sua concentração é estimada na saída. Este artigo propõe um sistema portátil de medida "on-line" feito através da variação da luz transmitida medida com um detector constituído por uma fonte de radiação visível e uma célula fotoelétrica e um software de coleta e tratamento de dados. Usando-se este sistema em uma extrusora de dupla rosca co-rotacional interpenetrante, pode-se verificar que a CDTR é muito mais sensível à variações na taxa de alimentação do que da velocidade de rotação da rosca e que as frações mais lentas do traçador podem demorar quatro vezes mais que as mais rápidas para saírem. Aumento na velocidade de rotação da rosca e/ou redução na taxa de alimentação aumenta o fluxo axial alargando a CDTR.

Palavras-chave: Tempo de residência, sistema de medida on-line, absorção de luz

\section{Introdução}

\section{A função distribuição de tempos de residência - FDTR}

O conhecimento do tempo que o material polimérico permanece dentro do equipamento durante o seu processamento é de fundamental importância ${ }^{[1,2]}$, principalmente quando se considera a grande sensibilidade destes materiais orgânicos à degradação termo-mecânica e mais recentemente nos casos onde a extrusora é utilizada como um reator, ou seja, na extrusão reativa. Nestes dois casos o tempo de permanência do polímero na extrusora é definido pelo compromisso de ser suficientemente longo para que todas as reações desejadas se completem e o mais curto possível para se evitar excessiva degradação do polímero. Durante o fluxo a massa fundida é trabalhada pela rosca subdividindo-a e forçando-a a mudar de direção inúmeras vezes. Tal comportamento é a principal característica de uma extrusora, o que garante a plastificação e mistura dos componentes. Este grande movimento forçado entre as cadeias poliméricas acaba por induzir diferentes velocidades em diferentes pontos da massa fundida, o que provoca o atraso ou adiantamento de algumas cadeias poliméricas ou partículas com relação à maioria

Denilson M. Santos,Universidade Federal do Maranhão, j1j1@zipmail.com.br e UFSCar/DEMa; Sebastião V. Canevarolo, Universidade Federal de São Carlos - UFSCar, Departamento de Engenharia de Materiais - DEMa, Rod. Washington Luís, Km 235, CEP 13565-905, Caixa Postal 676, São Carlos - SP. caneva@power.ufscar.br 
delas. Assim o tempo de residência não é um valor único mas uma distribuição em torno de um valor médio, com algumas poucas cadeias (partículas) saindo antes e outras saindo depois do denso conjunto que sai no tempo médio. Portanto o tempo de residência deve ser encarado como uma distribuição de tempos de residência, normalmente representada por uma função, denominada Função de Distribuição de Tempos de Residência - FDTR. Esta função é um importante parâmetro para a medida, caracterização e controle do fluxo em reatores e extrusoras. Sua forma indica o tipo de fluxo existente bem como variações no formato da curva podem mostrar alterações das condições de mistura, retenção de material em pontos mortos, etc. permitindo diagnósticos para um ajuste fino das condições de processamento.

\section{Obtenção da função distribuição de tempos de residência ${ }^{[3,4]}$}

Na maioria dos casos o fluxo é suficientemente complexo para praticamente impedir a obtenção da FDTR teoricamente, forçando o desenvolvimento e uso de técnicas experimentais para a sua determinação. A técnica mais difundida é a da "resposta a um estímulo pulsado" na qual, após a estabilização do processo, uma quantidade definida de um traçador (ou marcador) é instantaneamente introduzida no funil da extrusora (ou em outra entrada no cilindro) e em intervalos de tempo predefinidos coleta-se amostras na saída. Em cada amostra deste conjunto é determinada a concentração do traçador, através de uma técnica experimental apropriada, construindo-se o perfil de concentração, isto é a curva de concentração em função do tempo, $[\mathrm{C}(t)$ vs. $t]$. Inicia-se a contagem de tempo com $t=0$ no momento da adição do traçador e o tempo em que o primeiro vestígio do traçador sai da extrusora é chamado de tempo mínimo. A quantidade de traçador que sai do sistema depois do tempo mínimo define o seu perfil de distribuição na extrusora e é conhecido por Função da Distribuição de Tempos de Residência FDTR, representada matematicamente por $\mathrm{E}(t)$. Para facilitar os cálculos a área total sob a curva $[\mathrm{E}(t) \mathrm{vs} . t]$ é, por definição, igual a unidade. Mantendo-se a vazão da extrusora constante a quantidade de traçador, ou melhor sua concentração, pode ser medida de amostras retiradas em intervalos de tempo fixos $\Delta t$.
Portanto pode-se calcular o valor da função $E(t)$ em cada um destes intervalos através da divisão da concentração neste intervalo pela quantidade total de traçador introduzida ${ }_{0}^{\infty} C(t) \Delta t$, isto $\mathrm{e}^{[4]}$ :

$$
E(t)=\frac{C(t)}{{ }_{0}^{\infty} C(t) \Delta t}
$$

ou de maneira mais elegante, assumindo-se intervalos de tempo infinitesimais:

$$
E(t)=\frac{C(t)}{\int_{0}^{\infty} C(t) d t}
$$

onde $\mathrm{C}(t)$ agora representa o valor da concentração do traçador em um tempo $t$ qualquer.

Uma outra maneira de apresentação destes dados é na forma acumulativa onde o valor da função em um tempo $t$ qualquer é a concentração parcial acumulada de traçador que saiu até aquele instante. Esta função é conhecida como Função Acumulada da Distribuição do Tempo de Residência, FADTR representada matematicamente por $\mathrm{F}(t)$ e calculada como:

$$
F(t)=\int_{0}^{t} E(t) d t=\frac{0_{0}^{t} C(t) d t}{0_{0}^{\infty} C(t) d t}
$$

O cálculo do tempo de residência médio $\bar{t}$ pode ser obtido a partir das condições de conservação de massa, isto é:

$$
\bar{t}=\frac{V}{Q}=\frac{\text { volume }}{\text { vazão }}
$$

ou de maneira mais prática através da ponderação do tempo $t$ em qualquer intervalo $\Delta t$ com sua respectiva concentração de traçador $C(t) \Delta t$, isto é:

$$
\bar{t}=\frac{{ }_{0}^{\infty} t C(t) \Delta t}{{ }_{0}^{\infty} C(t) \Delta t}
$$

ou assumindo-se intervalos de tempo infinitesimais: 


$$
\bar{t}=\frac{\int_{0}^{\infty} t C(t) d t}{\int_{0}^{\infty} C(t) d t}
$$

e a variância $\sigma^{2}$ pode ser expressa por:

$$
\sigma^{2}={ }_{0}^{\infty}(t-\bar{t})^{2} E(t) \Delta t
$$

Uma maneira de reduzir o número de variáveis é trabalhar com o tempo de forma normalizada definido como:

$$
\theta=\frac{t}{t}
$$

assim as funções $\mathrm{E}(t)$ e $\mathrm{F}(t)$ podem ser reduzidas para $\mathrm{E}(\theta)$ e $\mathrm{F}(\theta)$ utilizando-se o tempo normalizado $\theta$ fazendo-se:

$$
\begin{aligned}
& \mathrm{E}(\theta)=t \mathrm{E}(t) \\
& \mathrm{F}(\theta)=\mathrm{F}(t)
\end{aligned}
$$

A fração retida de traçador $\mathrm{H}$ após o tempo de residência médio é definida por:

$$
H=1-\int_{0}^{\bar{t}} E(t) d t=1-\int_{0}^{\bar{t}} \frac{C(t)}{\int_{0}^{\infty} C(t) d t} d t
$$

os valores da fração retida estão na faixa de $0 \leq \mathrm{H} \leq 1$, para $\mathrm{H}=0$ tem-se fluxo tipo "plug", para $\mathrm{H}=0,368$ misturador ideal e $\mathrm{H}=1$ excessiva acumulação e tunelamento.

Devido a complexidade dos fluxos envolvidos nos sistemas reais, somente em alguns poucos casos foi possível desenvolver um equacionamento matemático para prever o fluxo. Segundo Levenspiel ${ }^{[5]}$ as funções do tipo FADTR para um misturador ideal e para um fluido da lei de potência em um reator tubular são respectivamente expressas pelas equações 11 e 12 .

$$
\begin{aligned}
& \mathrm{F}(\theta)=1-\mathrm{e}^{-\theta} \\
& F(\theta)=\left[1-\frac{n+1}{3 n+1} \frac{1}{\theta}\right]^{2 n / n+1} \cdot\left[1+\frac{2 n}{3 n+1} \frac{1}{\theta}\right] \\
& \text { para } \theta \geq \frac{\mathrm{n}+1}{3 \mathrm{n}+1}
\end{aligned}
$$

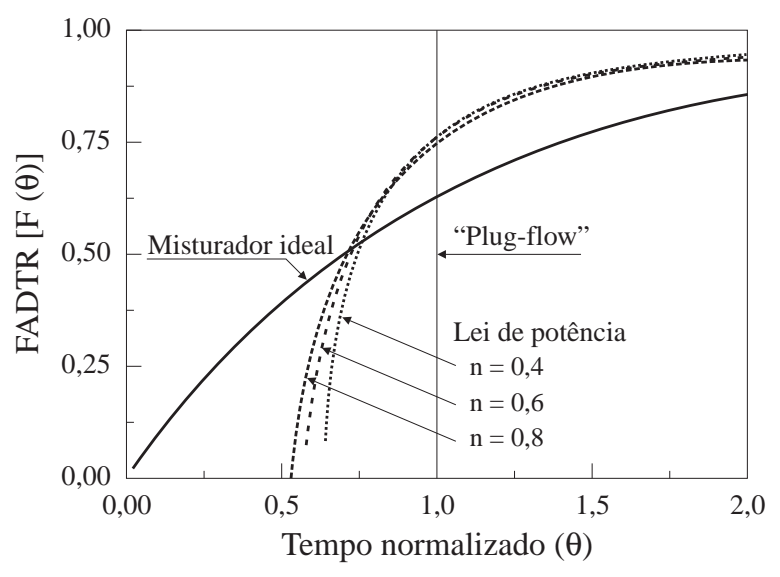

Figura 1. Comportamento característico da função FADTR $[\mathrm{F}(\theta)]$ para um misturador ideal, fluido da Lei de Potência para $\mathrm{n}=0,4 ; 0,6$ e 0,8$)$ e "Plug-flow".

A Figuralapresenta exemplos de funções acumulativas da distribuição de tempos de residência FADTR [ou $\mathrm{F}(\theta)$ ] no espaço do tempo normalizado $\theta$, mostrando uma curva de $\mathrm{F}(\theta)$ para o fluxo no misturador ideal calculada a partir da equação $11 \mathrm{e}$ outras três curvas referentes ao fluido lei das potências (equação 12), para diferentes índices de potência. Estas $F(\theta)$ se encontram no intervalo $0 \leq F(\theta) \leq 1$ e quando $\theta \rightarrow \infty, \mathrm{F}(\theta)=1$.

Como pode ser observado na Figura 1 as curvas calculadas são pouco afetadas por variações no índice de potência, mesmo que estas sejam significativas. Como valores práticos se encontram normalmente entre 0,36 a 0,38 é de se esperar pouca influência sobre a $F(\theta)$.

\section{Tipos de traçadores}

Em princípio o traçador deve ser uma molécula ou partícula que possa ser facilmente detectada e quantificada por alguma técnica experimental disponível e não deve alterar as características de fluxo da matriz. Em segundo lugar deve-se definir o que se pretende medir: se a distribuição de tempos de residência das cadeias poliméricas presentes no fluxo ou a capacidade de dispersão de uma partícula na massa polimérica fundida. No primeiro caso o melhor traçador a ser empregado é um formado pelo mesmo tipo de cadeia polimérica que o da matriz mas com algum ponto passível de identificação, por exemplo, um grupo lateral ou terminal graftizado. A presença deste grupo na massa polimérica poderá ser identificado e quantificado pela técnica experimental escolhida. Esta cadeia polimérica marcada deverá ser solúvel/miscível no polímero e apresentar propriedades reológicas semelhantes à matriz. No segundo caso, a partícula pode 
ser composta da própria carga a ser dispersa na massa. Em ambos os casos, vários tipos de traçadores podem ser utilizados dependendo basicamente da técnica de deteç̧ão e medida a ser utilizada. Historicamente o segundo tipo de traçador foi o mais utilizado e somente recentemente, com o advento de polímeros funcionalizados, cadeias poliméricas puderam ser marcadas e têm sido utilizadas.

Todd e Irving em $1969^{[6]}$ foram uns dos primeiros a usarem traçadores de nitrato de sódio e potássio para o estudo da mistura axial em misturadores/reatores com dupla rosca do tipo auto-limpante. Como fluido utilizaram uma solução de glicose e a detecção quantitativa era feita por medidas da condutividade de soluções aquosas das frações coletadas na saída da extrusora. Em 1975 Todd $^{[7]}$ propôs a utilização de corantes insolúveis (azul de metileno em polibuteno) que após extração das frações era quantificado por análise colorimétrica. Aproximadamente no mesmo período, Wolf \& White ${ }^{[3]}$ propuseram a utilização de traçadores radioativos, usando um concentrado com dióxido de manganês, que poderia ser irradiado em reator nuclear tanto antes da adição na extrusora como após a coleta das frações. Esta técnica continuou a ser utilizada por vários autores durante toda a década seguinte ${ }^{[4,8,9]}$. Potente e Lappe ${ }^{[10]} \mathrm{em} 1986$ desenvolveram um estudo comparativo entre corantes solúveis, pigmentos e microesferas de vidro, concluindo que o tipo de traçador tem uma profunda influência nas medidas da distribuição de tempos de residência. Alguns traçadores com intensa absorção na região do ultravioleta (mas fora da zona de absorção característica de anéis aromáticos monosubstituídos presentes em PS e tolueno) começaram a ser explorados a partir do início da década de 90, tais como: corante marrom avermelhado para poliestireno ${ }^{[11]}, 1$-aminoantraquinona para poliestireno ${ }^{[12,13]}$ e antracenometanol para EVA ${ }^{[14]}$. Também têm sido utilizadas cargas minerais do tipo microesferas de vidro, quantificadas por análise termogravimétrica ${ }^{[15]}$, dióxido de titânio, microesferas de vidro e carbonato de cálcio determinado por atenuação ultra-sônica ${ }^{[16,17]} \mathrm{e}$ cargas metálicas do tipo flocos de alumínio utilizados em EVA e quantificados por dissolução e extração em clorofórmio $^{[18]}$. Poucos traçadores com características de absorção na região do infravermelho têm sido usados sendo um deles o tiocianato de potassio $\mathrm{KSCN}$ que pode ser quantificado pela sua absorção a $2056 \mathrm{~cm}^{-1}$ [19] . Poucos trabalhos utilizam a absorção de luz na região do visível como detetores empregando negro de fumo como traçador em polipropileno ${ }^{[20]}$.

A produção de cadeias poliméricas marcadas é um desenvolvimento bastante recente, tendo como primeiro caso a graftização de antraceno sobre cadeias de poliestireno funcionalizado com grupos isocianatos ${ }^{[21,22]}$. Tal PS marcado com antraceno, quantificado por espectroscopia de ultravioleta, foi utilizado como traçador para a determinação das funções de tempo de residência de poliestireno.

Medidas off-line demandam um tempo muito longo e não correspondem à velocidade de resposta requerida pelas necessidades atuais. Recentemente ${ }^{[23]}$ tem-se notado um crescente interesse no desenvolvimento de técnicas on-line com resposta em tempo real, utilizando-se espectroscopia de infravermelho (tanto MID quanto NEAR-IR), ressonância magnética nuclear, e métodos ultra-sônicos para a caracterização do produto e controle do processo. A partir da segunda metade da década de 90 várias técnicas quantitativas para determinação da distribuição do tempo de residência têm sido desenvolvidas com detecção on-line ou em tempo real do traçador por espectroscopia de ultravioleta $^{[21,22]}$, infravermelho ${ }^{[22,24]}$, métodos ultrasônicos (velocidade e atenuação) ${ }^{[16,17]}$ e sistemas com absorção de luz por parte do traçador ${ }^{[20,25]}$, onde, a passagem do mesmo absorve parte do feixe luminoso, sendo tal absorção quantificada. Técnicas on-line não só reduzem o tempo de obtenção do resultado experimental mas principalmente aumentam a velocidade de decisão pois fornecendo resultados em curtos intervalos de tempo (minutos comparados com vários dias quando feito pelo método convencional off-line) permitem sua utilização para fazer finos ajustes durante $o$ processamento comercial. Também deve-se considerar a eliminação do trabalhoso método de coleta e posterior análise, o que normalmente restringe em muito o número de frações por amostra, aumentando a incerteza na definição da curva da FDTR.

Este artigo descreve os resultados obtidos com a medida da distribuição de tempos de residência, durante a extrusão de um polímero, a partir da coleta de dados com um sistema portátil de medida online, onde o detector usa radiação visível e não precisa tocar a amostra.

\section{Procedimento experimental}

\section{Materiais}

Para as medidas de extrusão usou-se polipropileno grau extrusão da OPP $\left(\rho=0.90 \mathrm{~g} / \mathrm{cm}^{3}\right.$ e MFI $=$ 
$16 \mathrm{~g} / 10 \mathrm{~min})$. Como traçador foi usado um concentrado de cor vermelho na forma de grãos (produto comercial da CROMEX ), composto basicamente por um pigmento vermelho sem cobertura (isto é, não possui $\mathrm{TiO}_{2}$ ) disperso em uma matriz de EVA. Para comparação também usou-se tiocianato de potássio (KSCN) como traçador ${ }^{[19]}$ retirando-se amostras a cada $5 \mathrm{seg}$.

\section{Métodos}

\section{Equipamentos}

Recentemente desenvolvemos um completo sistema compacto e portátil para a obtenção das curvas FDTR e de todas as demais variáveis características de tais curvas incluindo-se, tempo de residência mínimo, médio, máximo, fração retida, variância, etc. Este é constituído de um detector por absorção de luz visível e uma interface de coleta e transferência de dados a um computador portátil. O diagrama esquemático de tal sistema é apresentado na Figura 2. Todos os cálculos e apresentação das curvas na tela são feitos por um programa especialmente desenvolvido para este fim. O tempo necessário para coleta dos dados, cálculo e apresentação das curvas em tela é menor que o tempo de residência do polímero na extrusora ${ }^{[26]}$.

$\mathrm{O}$ detector tem uma caixa de controle com dois longos braços metálicos flexíveis em cujas pontas se encontram uma lâmpada de filamento e uma célula fotoelétrica. A fonte de luz é posicionada ao lado do extrudado em forma de fio circular, o mais próximo possível da saída da extrusora, permitindo que o feixe de luz atravesse o polímero fundido. A passagem do traçador diminui a intensidade de luz transmitida, que vai sendo monitorada ao longo do tempo do outro lado do extrudado pela célula fotoelétrica, efeito esse, que descreve a curva de distribuição do traçador na massa polimérica.
Na caixa de controle existem dois potenciômetros para o ajuste do zero e do ganho, permitindo o controle da intensidade e sensibilidade do sinal. O sinal analógico é coletado por uma interface externa DAQ-Pad 1200 , a uma taxa de 20 pontos por segundo, e enviada para o computador via a saída paralela da impressora. Dos vários canais que esta interface possui apenas dois são usados: um para receber o sinal do detector e outro para o sinal do botão de partida.

O software especialmente desenvolvido coleta os sinais e calcula todas as curvas. Este consiste de quatro módulos:

i) Linha de base; onde o sinal do detector (ou outro canal qualquer a ser especificado) é apresentado em tela de maneira contínua para permitir o controle visual do funcionamento do sistema, estabilização do fluxo na extrusora e da própria linha de base de leitura.

ii) Amostra; onde todos os dados da amostra (tipo de polímero e traçador) e as condições de medida (perfil de temperatura da extrusora, velocidade de rotação da rosca e de alimentação dos componentes, configuração da rosca, tempo de medida total e canais de leitura, etc.) são digitados e armazenados.

iii) Medidas on-line; mostra no visor a curva que se esta obtendo, tempo de medida e valor do sinal lido (em $\mathrm{mV})$, botões para término e salvamento dos dados captados, etc. A aquisição dos dados pode ser iniciada através de pressão manual no botão de partida, ligado ao segundo canal, no exato momento em que o traçador é introduzido na extrusora.

iv) Análise; calcula e mostra na tela as várias curvas, tais como: tempo de residência (CDTR), função distribuição do tempo de residência (FDTR), função acumulativa da distribuição de tempos de residência (FADTR) e diferença en-

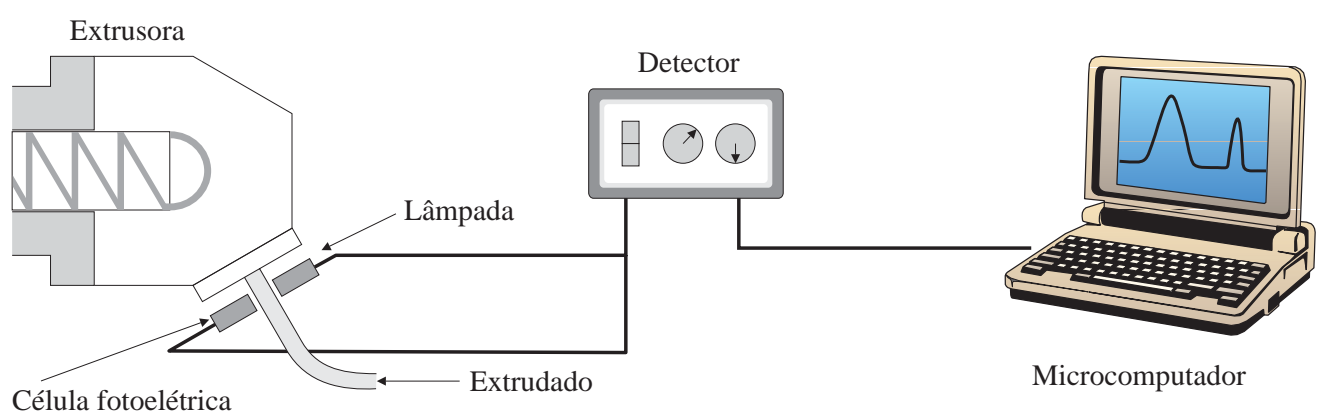

Figura 2. Diagrama esquemático do sistema proposto para medida on-line da Distribuição de Tempos de Residência durante a extrusão. 
tre duas FADTRs. Todos os dados podem ser armazenados para serem analisados em outros softwares disponíveis comercialmente ( por ex. Origin, Excel, Statistics, etc.). Para comparação dos resultados obtidos também está disponível um banco de dados com várias curvas padrões CDTR e FADTR simétricas, assimétricas como também calculadas através da equação da lei de potência.

\section{Medidas "ON-LINE"}

As medidas da distribuição de tempos de residência do fundido polimérico foram feitas em uma extrusora de dupla rosca Werner-Pfleiderer ZSK 30 com dosagem controlada por um alimentador gravimétrico K-Tron, para manter a taxa de alimentação constante, permitir um fluxo estável e um perfil de temperatura de: $190 ; 200 ; 210 ; 210 ; 200^{\circ} \mathrm{C}$ nas seguintes condições de operação; Rotação: 100, 150 e 200rpm, e Taxa de Alimentação: 5; 7,5 e 10 $\mathrm{kg} / \mathrm{h}$. Os dados são comparados com algumas curvas teóricas

\section{Quantificação do traçador por espectroscopia no infravermelho}

Amostras contendo tiocianato de potássio (KSCN) como traçador coletadas em intervalos fixos de tempo foram prensadas a $190^{\circ} \mathrm{C}$, em uma prensa hidráulica com pratos aquecidos, formando filmes de aproximadamente $50 \mu \mathrm{m}$. Utilizando-se espectroscopia de infravermelho calculou-se a concentração de traçador através da relação das absorbâncias a 2056 $\mathrm{cm}^{-1}$ (característica do grupo $\mathrm{CN}$ ) e a $1170 \mathrm{~cm}^{-1}$ (característica do polipropileno).

\section{Resultados e Discussão}

A Figura 3 mostra a distribuição de pontos experimentais coletados durante a medida da Curva de Distribuição de Tempos de Residência (CDTR) do fundido polimérico na extrusão, sob uma taxa de alimentação de $7,5 \mathrm{~kg} / \mathrm{h}$ e uma velocidade de rotação de rosca de $100 \mathrm{rpm}$. Nota-se algum espalhamento dos resultados, mas este é aleatório em torno de um valor médio. Isto se deve a medida a ser feita em um ambiente iluminado com luz artificial fluorescente, ciclando a $60 \mathrm{~Hz}$, valor próximo à

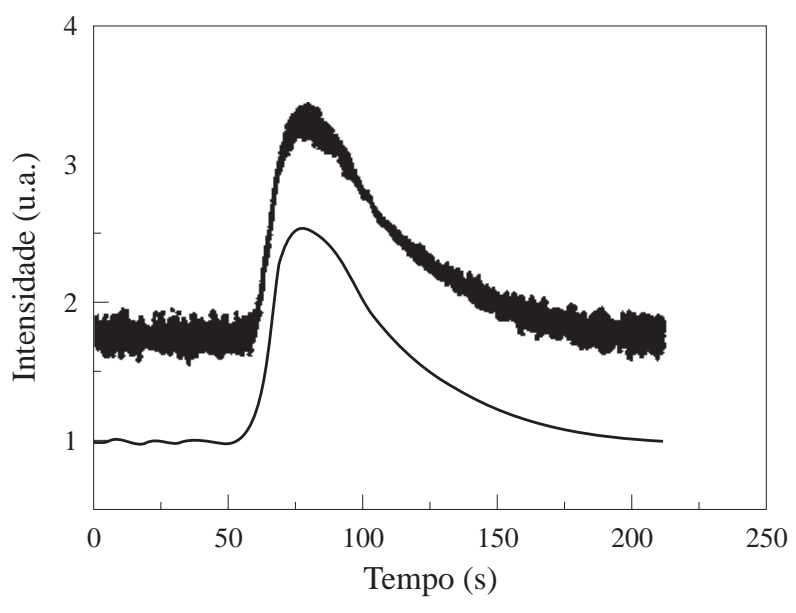

Figura 3. Efeito do alisamento da curva fazendo-se uma média com os 50 pontos adjacentes. As curvas estão deslocadas para facilidade de visualização.

freqüência de coleta de dados $(20 \mathrm{~Hz})$. Fazendo-se um alisamento matemático, do tipo média dos 50 pontos adjacentes, obtém-se a segunda curva, mostrada na mesma figura. Tal procedimento não afeta os resultados e portanto foi considerado padrão e utilizado em todos os gráficos apresentados neste trabalho.

Em função das especificações do sistema aqui proposto a coleta de dados pode ser feita a uma freqüência máxima de $20 \mathrm{~Hz}$, ou seja uma leitura a cada $50 \mathrm{~ms}$. Tal velocidade de aquisição é suficientemente alta para gerar curvas contínuas, permitindo-se uma fácil detecção, mesmo de pequenas variações no seu perfil, aumentando o poder de análise. Esta freqüência foi definida como padrão e usada em todas as medidas apresentadas neste trabalho.

Para a determinação da quantidade ideal de traçador a ser usado nas medidas obteve-se curvas CDTR com

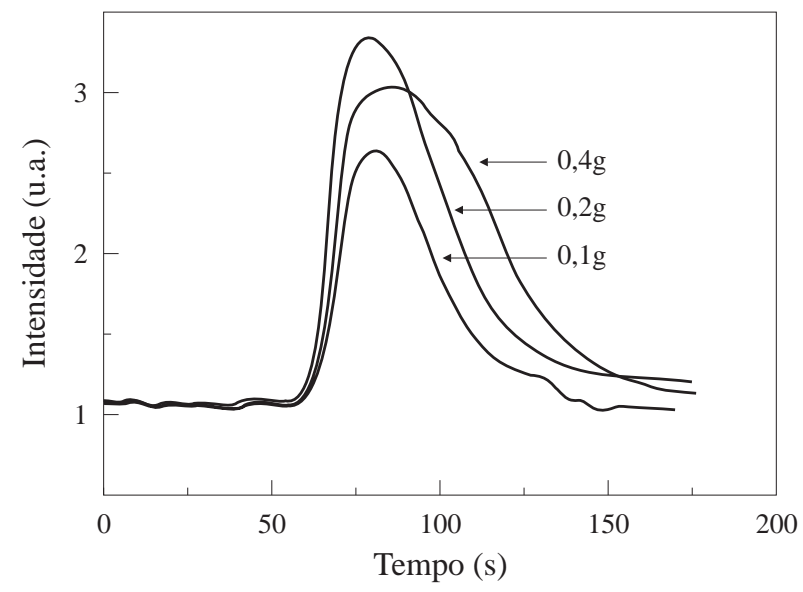

Figura 4. Efeito da concentração do traçador na CDTR medidos em uma extrusora de rosca dupla. 
Tabela 1. Parâmetros médios medidos ou calculados segundo as equações anteriores:

\begin{tabular}{ccccc}
\hline $\begin{array}{c}\text { Peso do traçador por pulso } \\
(\mathbf{g})\end{array}$ & $\boldsymbol{t}_{\mathbf{i}}(\mathbf{s})$ & $\boldsymbol{t}_{\mathbf{f}}(\mathbf{s})$ & $\overline{\boldsymbol{t}}(\mathbf{s})$ & $\boldsymbol{H}$ \\
\hline 0,1 & 58 & 161 & 80 & 0,436 \\
0,2 & 58 & 164 & 79 & 0,498 \\
0,4 & 59 & 173 & 91 & 0,463 \\
\hline
\end{tabular}

quantidades crescentes variando-se de $0,1 \mathrm{~g}$ a $0,4 \mathrm{~g}$ por pulso, ou seja, a quantidade de traçador introduzida no funil de alimentação. A Figura 4 mostra este efeito na forma da curva e consequentemente nos resultados medidos do tempo inicial $t_{i}$ (tempo para aparecer a primeira fração do traçador), final $t_{f}$ (tempo para sair a última fração do traçador), médio $t$ e fração retida $H$,os quais são apresentados na Tabela 1.

Como esperado o valor do tempo inicial $t_{i}$ não é afetado pela concentraçãa estando a variação de leituras dentro do erro de medida ( $\pm 1 \mathrm{seg})$. Por outro lado o valor final $t_{f} \mathrm{o}$ é, sendo maior quanto maior for a quantidade do traçador usado, devido ao alargamento do pulso inicial. Outro problema mais grave das altas concentrações é a saturação do detector que tende a perder a linearidade entre concentração e intensidade do sinal, necessidade básica para operação do detector. A fração retida de traçador $(H)$ após o tempo de residência médio não é substancialmente afetada pela concentração do traçador. Calculando-se os tempos médios $\bar{t}$ podese observar que estes são muito próximos enquanto o detetor não satura (até $0,2 \mathrm{~g}$ de traçador por pulso), ou seja, o valor mais conveniente para as condições usadas foi de $0,1 \mathrm{~g}$ de traçador por pulso. Assim, definiu-se como padrão esta concentração, usando-a para todas as outras medidas apresentadas neste trabalho, independentemente das condições de processamento.

A Figura 5 apresenta curvas de distribuição de tempo de residência, para uma rotação de $200 \mathrm{rpm}$ e taxa de alimentação de $10 \mathrm{Kg} / \mathrm{h}$, obtidas pela técnica aqui proposta comparadas às obtidas por espectroscopia de infravermelho, técnica experimental comum na literatura ${ }^{[19]}$. A primeira curva foi obtida segundo a metodologia proposta neste trabalho utilizando-se como traçador o concentrado vermelho. A segunda curva, com um número bem menor de pontos, corresponde aos resultados utilizando-se tiocianato de potássio como traçador, tendo sua concentração estimada através de espectroscopia de infravermelho. Como o KSCN

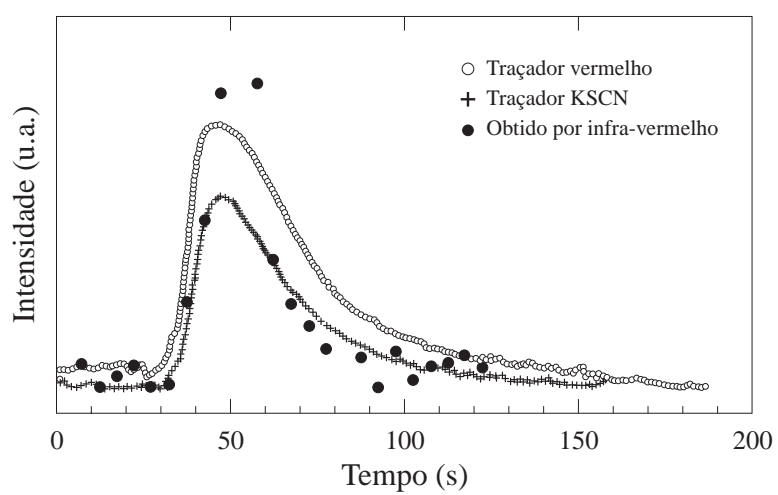

Figura 5. Comparação das medidas da CDTR segundo a técnica aqui apresentada, utilizando-se um traçador de concentrado vermelho(o), $\mathrm{KSCN}(+)$, e por espectroscopia de infravermelho $(\bullet)$.

altera as características ópticas do polímero fundido também foi possível detectá-lo pelo sistema aqui proposto, obtendo-se a terceira curva. Como a intensidade tem unidade arbitrária pode-se concluir que todas as curvas são equivalentes, validando a técnica proposta. A enorme diferença do número de pontos entre as duas técnicas utilizadas permite também uma maior confiança nos resultados, principalmente na região caudal da curva.

A seguir estudou-se a influência da taxa de alimentação e da velocidade de rotação da rosca sobre a CDTR, em uma extrusora de dupla rosca corotacional interpenetrante. Estas são mostradas nas Figuras 6 e 7.

A Figura 6 mostra a influência da taxa de alimentação, variando entre 5 e $10 \mathrm{~kg} / \mathrm{h}$, para valores constantes da velocidade de rotação da rosca (100 a 200 rpm) na Distribuição de Tempos de Residência. A Figura 7 mostra as mesmas curvas para valores constantes da taxa de alimentação. Os valores numéricos mais importantes calculados destas curvas se encontram apresentados na Tabela 2. Todas as cur-

Tabela 2. Efeito da velocidade de rotação da rosca (rpm) e da taxa de alimentação $(\mathrm{kg} / \mathrm{h})$ nos parâmetros médios de tempos de residência na extrusora de rosca dupla ZSK-30.

\begin{tabular}{cccccc}
\hline $\begin{array}{c}\text { Corrida } \\
(\mathbf{r p m} / \mathbf{k g} / \mathbf{h})\end{array}$ & $\boldsymbol{t}_{\boldsymbol{i}}(\mathbf{s})$ & $\boldsymbol{t}_{f}(\mathbf{s})$ & $\boldsymbol{t}_{p}(\mathbf{s})$ & $\overline{\boldsymbol{t}}(\mathbf{s})$ & $\boldsymbol{H}$ \\
\hline $100 / 5,0$ & 70,7 & 216,4 & 103,9 & 122,7 & 0,439 \\
$100 / 7,5$ & 57,1 & 205,3 & 76,2 & 97,5 & 0,410 \\
$100 / 10$ & 45,8 & 139,7 & 62,6 & 74,1 & 0,421 \\
$150 / 5,0$ & 62,9 & 215,3 & 90,8 & 111,6 & 0,434 \\
$150 / 7,5$ & 48,8 & 208,6 & 70,2 & 82,3 & 0,426 \\
$150 / 10$ & 38,8 & 155,5 & 54,8 & 71,0 & 0,388 \\
$200 / 5,0$ & 53,3 & 200,0 & 84,2 & 102,5 & 0,439 \\
$200 / 7,5$ & 41,7 & 148,6 & 63,3 & 77,0 & 0,423 \\
$200 / 10$ & 33,9 & 124,5 & 50,7 & 62,6 & 0,422 \\
\hline
\end{tabular}




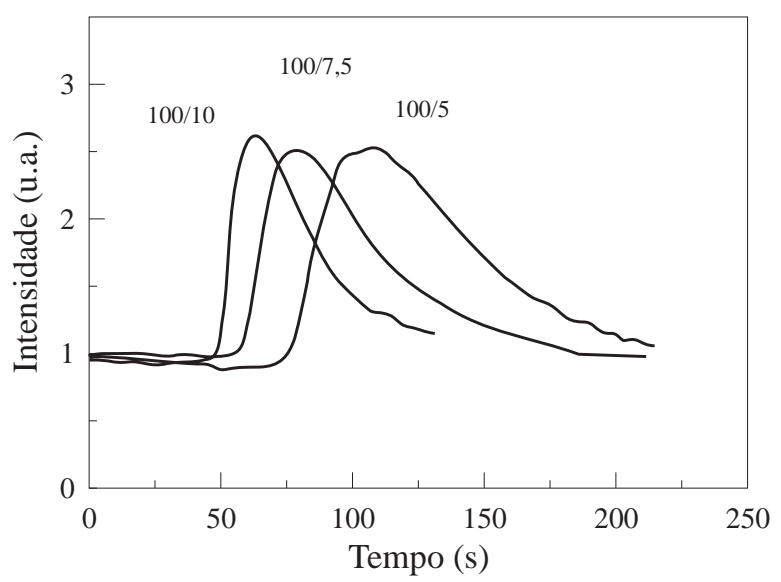

6(a): Velocidade de rotação de rosca fixa em $100 \mathrm{rpm}$.

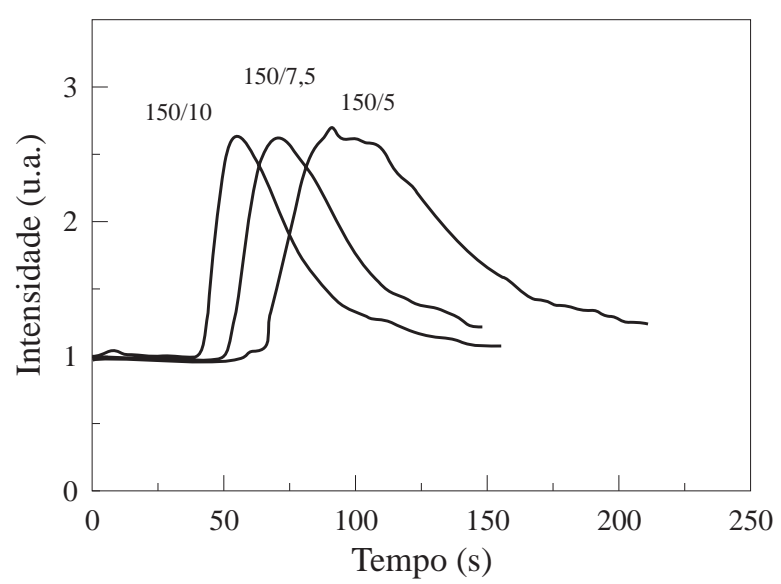

6(b): Velocidade de rotação de rosca fixa em $150 \mathrm{rpm}$.

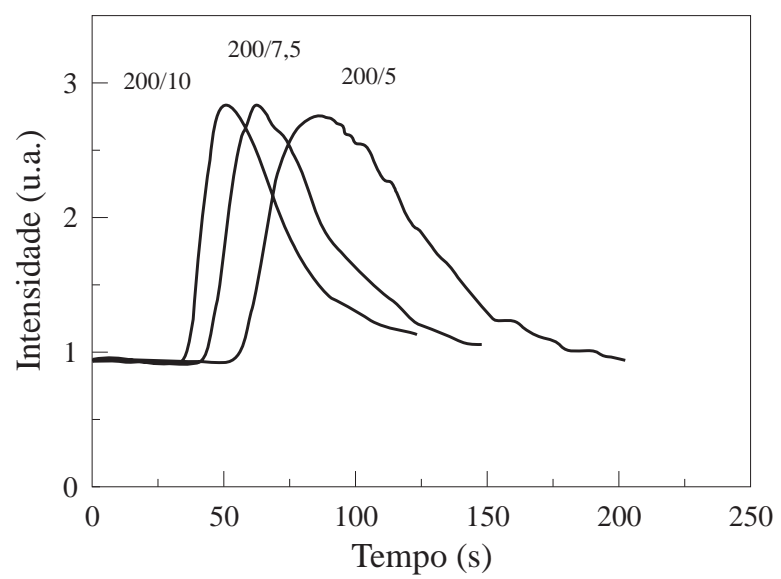

6(c): Velocidade de rotação de rosca fixa em $200 \mathrm{rpm}$.

Figura 6. Influência da rotação de rosca (rpm) e da taxa de alimentação $(\mathrm{kg} / \mathrm{h})$ na Extrusora ZSK-30 sobre a CDTR.

vas apresentam a forma característica com uma distribuição assimétrica em torno de um valor médio. $\mathrm{O}$ valor médio té aproximadamente $15 \%$ acima do valor de pico $t_{p}$ (tempo relativo à maior intensidade nas curvas de distribuição de tempos de residência), bem como o valor de tempo final $t_{f}$ é aproximadamente quatro vezes

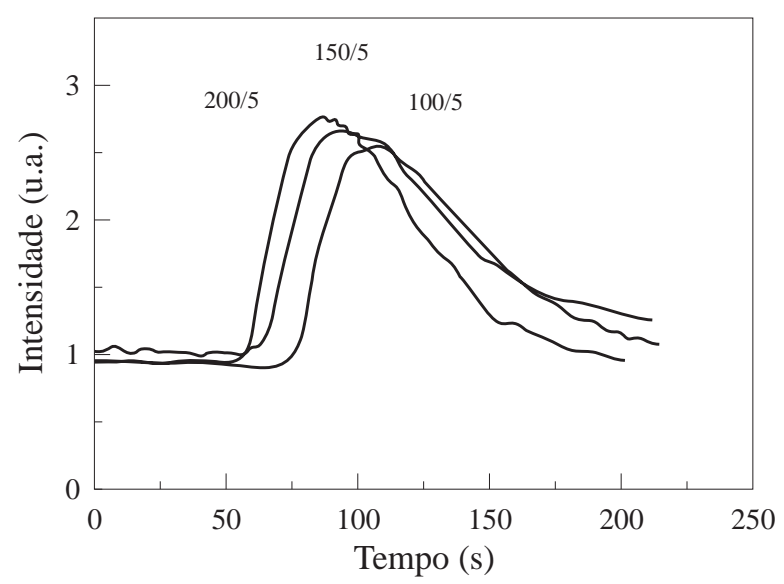

7(a): Taxa de alimentação fixa em $5,0 \mathrm{~kg} / \mathrm{h}$.

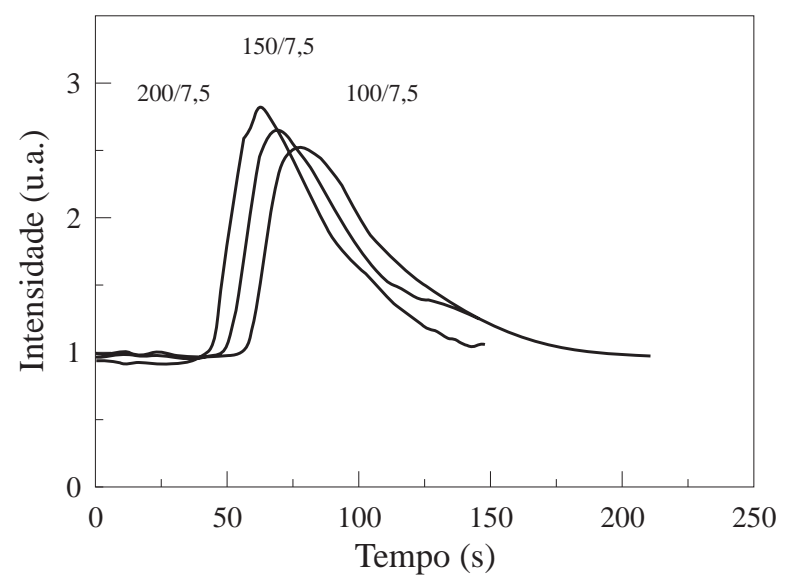

7(b): Taxa de alimentação fixa em $7,5 \mathrm{~kg} / \mathrm{h}$.

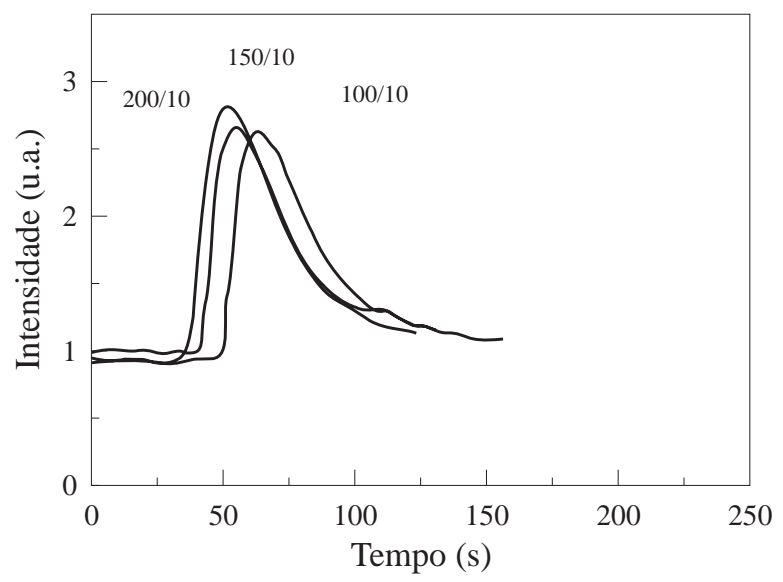

7(c): Taxa de alimentação fixa em $10,0 \mathrm{~kg} / \mathrm{h}$.

Figura 7. Influência da rotação de rosca (rpm) e da taxa de alimentação $(\mathrm{kg} / \mathrm{h})$ na Extrusora ZSK-30 sobre a CDTR.

maior que o valor do tempo inicial $t_{i}$, ou seja, as frações mais lentas podem demorar até quatro vezes mais que as mais rápidas para serem bombeadas para fora da extrusora. Tanto um aumento da velocidade de rotação da rosca quanto da taxa de alimentação tende a encolher a curva de distribuição de tempo de residência, deslo- 
cando-a para a esquerda, reduzindo todos os seus valores médios de tempo $\left(t_{i}, t_{p}, t_{f}\right.$ e $\left.t\right)$. O efeito é muito maior para a taxa de alimentação mostrando que a CDTR é muito mais sensível à mudanças nesta variável do que na velocidade de rotação da rosca. A fração retida de traçador $(H)$ é levemente reduzida com a aumento da taxa de alimentação pois quanto maior a alimentação maior é o preenchimento da rosca, principalmente próxima a regiões de alta pressão e portanto menor a presença de fluxo axial. Esta variável praticamente não é afetada pela velocidade de rotação da rosca. Além de quantificar o processo de extrusão estes dados também podem ser usados para ajustar as condições de processamento on-line e ajudar durante o projeto do perfil da rosca.

A Figura 8 mostra os mesmos dados das Figuras 6 e 7 apresentados em termos da Função Acumulativa da Distribuição de Tempos de Residência FADTR. Em termos gerais observa-se que o comportamento do fluxo em uma extrusora de rosca dupla tende para o "plug-flow", com pouco fluxo axial, como já observado por outros autores ${ }^{[2,10]}$. As curvas são bastante próximas e para evitar dificuldade na sua visualização apenas três condições limites são apresentadas. A curva referente à condição de mínima velocidade de rotação da rosca e máxima taxa de alimentação (100/10), ou seja, rosca cheia e em baixa rotação, é a que mais se aproxima do comportamento "plug-flow". Um gradual aumento na rpm e/ou redução na alimentação tendem a aumentar o fluxo axial alargando a distribuição, como também previsto pela fração retida de

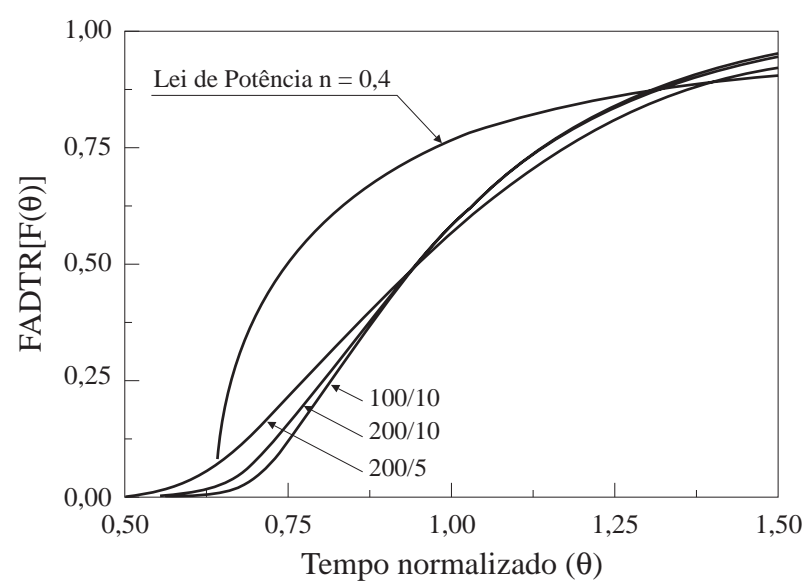

Figura 8. Idem à Figura 7 mas com relação à Função Acumulativa da Distribuição de Tempos de Residência FADTR. Para comparação também é apresentada a curva calculada para um polímero com fluxo segundo a lei de potência (para $n=0,4$ ). Observar a expansão da escala do tempo normalizado $\theta$. traçador $(H)$, discutida acima. Assim a condição 200/5 é a que mais se afasta do comportamento "plug-flow", sendo as demais intermediárias. Apenas como referencia é apresentado novamente a curva teórica mostrada na Figura 1 para um fluido que segue a Lei de Potência $\operatorname{com} \mathrm{n}=0,4$.

\section{Conclusões}

O sistema de medida on-line por absorção de luz no visível proposto detecta de maneira muito conveniente o Tempo de Residência em extrusoras. O software desenvolvido permite o cálculo imediato de todas as curvas relativas às Funções Acumulativa e de Distribuição de Tempos de Residência FADTR e FDTR respectivamente, bem como todos os parâmetros relacionados às distribuições $\left(t_{i}, t_{p}, t, H\right)$. O sistema é compacto, leve e portátil, obtém a medida sem a necessidade de tocar a amostra, fazendo-o de modo fácil e rápido. Usando-se este sistema em uma extrusora de dupla rosca interpenetrante co-rotacional pode-se verificar que a CDTR é muito mais sensível à variações na taxa de alimentação do que da velocidade de rotação da rosca. Também observa-se que as frações mais lentas podem demorar quatro vezes mais que as mais rápidas para saírem. Um aumento na velocidade de rotação da rosca e/ou redução na taxa de alimentação, ou seja uma rosca pouco preenchida e em alta rotação, tende a aumentar o fluxo axial alargando a distribuição de tempo de residência.

\section{Agradecimentos}

Os autores agradecem a participação do Sr. Gil Mason pela construção do detetor e ao Sr. Sérgio R. Barcellos por escrever o software, bem como ao suporte financeiro do CNPq (PADCT, PRONEX) CAPES/PICD e FAPESP em vários projetos.

\section{Referencias bibliográficas}

1. Carberry, J.; Varma, A., Chemical Reaction and Reactor Engineering. Ed. Marcel Dekker, Inc. New York, (1986).

2. Tadmor, Z; Gogos, C. G., Principles of Polymer Processing; Wiley Intercience, New York, (1978). 
3. Wolf, D., White, D. H. - AIChE. J., 22, 1, 122-131 (1976).

4. Wolf, D., Holin, N., White, D. H. - Polym. Eng. Sci., 26, 9, 640-646 (1986).

5. Levenspiel, O. Chemical Reaction Engineering. $2^{\circ}$ Ed., Wiley, New York, (1972).

6. Todd, D. B.; Irving, H. F.- Chem. Eng. Prog. 65, 9, 84-89 (1969).

7. Todd, D. B. - Polym. Eng. Sci., 15, 6, 437-443 (1975).

8. Janssen, L. P. B. M.; Hollander, R. W.; Spoor, M.W.; Smith, J., M..AIChE J. 25(2), 345-351 (1979).

9. Tzoganakis, C., Tang, Y., Vlachopoulos, J., Hamielec, A. E. - J. Appl. Polym. Sci., 37, 681693 (1989).

10. Potente, H., Lappe, H. - Plast. Rubb. Proc. Appl., 6, 2, 135-140 (1986).

11. Chen, L.; Pan, Z. - J. Chem. Ind. Eng. China, 5, 2, 153-159 (1990).

12. Chen, L., Pan, Z., Hu, G.-H., AIChE. J., 39, 9, 1455-1464 (1993).

13. Chen, L., Hu, G. H., Lindt, J. T. - Polym. Eng. Sci. 35, 7, 598-603 (1995).

14. Oberlehner, J., Cassagnau, P., Michel, A. Chem. Eng. Sci., 49, 23, 3897-3907 (1994).

15. Weiss, R. A., Stamato, H. - Polym. Eng. Sci., 29, 2, 134-139 (1989).
16. Gendron, R.; Dumoulin, M. M.; Tatibouet, J.; Piche, L.; Hamel, A., - Anais da Soc. Plast. Eng. SPE ANTEC'93, 2256-2261 (1993).

17. Huneault, M. A. - Anais da Soc. Plast. Eng. SPE - RETEC POLYBLENDS'97, 165-187, 9-10/ Out, Canada, (1997).

18. Kim, P. J., White, J. L. - Intern. Polym. Proc., IX, 2, 108-118, (1994).

19. Vainio, T. P., Harlin, A., Seppälä, J. V. - Polym. Eng. Sci. 35, 3, 225-232 (1995).

20. Chen, T.; Patterson, W. I.; Dealy, M. J., Intern. Polym. Proc. X , 1, 3-9 (1995).

21. Hu, G. H., Kadri, I., Anais da Polym. Proc. Soc. PPS, 19-21/Ago, Sweden (1997).

22. Nietsch, T., Cassagnau, P., Michel, A, - Anais do Polym. Proc. Soc. PPS-13, 11-J, Secaucus, N. J., 10-13/Jun, USA (1997).

23. Patel, S. H., Todd, D. B., Xantos, M. - Anais da Soc. Plast. Eng. SPE ANTEC'94, 22142219 (1994).

24. Fischer, T. Bayer, L. Jakisch, M. Stephan, K-J. Eichhorn,; Anais do Polym. Proc. Soc. PPS-13, 11-F, Secaucus, N. J., 10-13/Jun, USA (1997).

25. Potente, H.; Koch, M., - Advan. Pol. Technol. 9, n 2, 119 - 127 (1989).

26. Santos, D. M., Canevarolo, S. V. - Anais do 13 CBECIMAT, 4166-4175, 6-9/Dez, Curitiba / PR (1998).

Recebido em:20/10/98 Aprovado em: 10/06/99 